\title{
Nutrient stoichiometry shapes microbial coevolution
}

\author{
Megan L. Larsen ${ }^{1}$, Steven W. Wilhelm², Jay T. Lennon*1
}

4

${ }^{1}$ Department of Biology, Indiana University, Bloomington, IN 47405

6

${ }^{2}$ Department of Microbiology, University of Tennessee, Knoxville, TN 37996

*Correspondence to: lennonj@indiana.edu.

8

Running title: Nutrient stoichiometry and coevolution

10 Keywords: bacteria, phage, resources, resistance, arms race, networks

Article type: Letter

12 Abstract word count:

(original manuscript: 150; revised manuscript: 150)

14 Main text word count: (original manuscript: 4817; revised manuscript: 5369)

Reference count: (original manuscript: 50; revised manuscript: 52)

16

Figure count: 5

18 Table count: 0

20 Author contributions: MLL, SWW, and JTL designed study; MLL performed research; MLL and JTL analyzed data; MLL, SWW, and JTL wrote paper.

Available code and data. Data and code are available in the public GitHub repository

24 https://github.com/LennonLab/eco-evo-stoich 


\section{ABSTRACT}

Coevolution is a force contributing to the generation and maintenance of biodiversity. It

28 is influenced by environmental conditions including the scarcity of essential resources, which can drive the evolution of defense and virulence traits. We conducted a long-term chemostat

30 experiment where the marine cyanobacterium Synechococcus was challenged with a lytic phage under nitrogen $(\mathrm{N})$ or phosphorus $(\mathrm{P})$ limitation. This manipulation of nutrient

32 stoichiometry altered the stability of host-parasite interactions and the underlying mode of coevolution. By assessing infectivity with $>18,000$ pairwise challenges, we documented

34 directional selection for increased phage resistance, consistent with arms-race dynamics while phage infectivity fluctuated through time, as expected when coevolution is driven by

36 negative frequency-dependent selection. The resulting infection networks were $50 \%$ less modular under $\mathrm{N}$ - versus P-limitation reflecting host-range contraction and asymmetric 38 coevolutionary trajectories. Nutrient stoichiometry affects eco-evolutionary feedbacks in ways that may alter the dynamics and functioning of environmental and host-associated 40 microbial communities. 


\section{INTRODUCTION}

In order to grow and reproduce, organisms must assimilate resources from the

environment to meet their nutritional and energetic demands. Ecological stoichiometry is a

theoretical framework that explicitly considers the mass balance of materials and energy in the

environment and the individuals that incorporate them (Sterner \& Elser 2002). Of the

52 approximately 25 elements contained in biomass, nitrogen $(\mathrm{N})$ and phosphorus $(\mathrm{P})$ are two of the most limiting and essential nutrients. $\mathrm{N}$ and $\mathrm{P}$ are needed for the synthesis of major

54 macromolecules, including nucleic acids, ribosomes, proteins, and cellular membranes that collectively influence an organism's performance. However, the degree to which $\mathrm{N}$ and P are

56 regulated differs among major groups of taxa. For example, the biomass stoichiometry of primary producers tends to be flexible, reflecting the supply of nutrients in their environment

58 (Sterner \& Elser 2002). In contrast, the biomass stoichiometry of consumer populations (e.g., viruses, invertebrates, mammals) is homeostatically regulated and therefore tends to remain constant even when the nutrient content of their resources fluctuates (Sterner \& Elser 2002).

Such differences can create a nutritional imbalance between primary producers and consumers,

62 which has profound consequences for a wide range of ecological processes including resource competition, host-parasite dynamics, and ecosystem functioning (Smith \& Holt 1996; Hall 2009;

64 Aalto et al. 2015).

Nutrient stoichiometry can also influence evolutionary processes. Nutrient limitation

66 often leads to a reduction in population size, which can diminish the efficiency of selection and result in the accumulation of deleterious mutations through genetic drift (Kimura 1962).

68 However, many populations have adaptations that allow individuals to contend with absolute and relative resource scarcity. For example, the disproportionate use of nucleotides that vary in $\mathrm{N}$ 
70 content reduces stoichiometric mismatch between an organism and its environment (Elser et al. 2011). Similarly, natural selection can operate on the material costs of gene expression in ways

72 that lead to the sparing of elements that are normally used in highly expressed proteins (Bragg \& Wagner 2009). These genetic responses to nutrient limitation may give rise to the evolution of

74 organismal stoichiometry. For example, long-term carbon limitation led to increased $\mathrm{N}$ and $\mathrm{P}$ content of experimentally evolved bacterial populations (Turner et al. 2017). However,

76 evolutionary responses to nutrient stoichiometry may depend on the identity of the limiting nutrient (Gresham et al. 2008). While N limitation can select for stress-related or catabolic genes

78 with lower guanine-cytosine content (Acquisti et al. 2009), P limitation can favor the replacement of phospholipids with sulfolipids in the cell membranes of certain microorganisms

80 (Van Mooy et al. 2006).

When considering multiple interacting species, the combined effects of nutrient

82 stoichiometry may give rise to eco-evolutionary feedbacks. Such feedbacks occur when ecological interactions affect evolutionary processes, which in turn modify species interactions

84 and ecological dynamics (Haloin \& Strauss 2008). For example, the rapid evolution of functional traits can produce diminished oscillations, longer periods of cycling, and phase-shifted

86 population densities between hosts and their parasites, a phenomenon referred to as "cryptic dynamics" (Yoshida et al. 2007). Theory suggests that cryptic dynamics can arise when nutrient

88 stoichiometry alters the stability of antagonistic species interactions (Yamamichi et al. 2015), which may ultimately intensify the modes of coevolution, namely arms-race dynamics and

90 negative frequency-dependent selection (Aalto et al. 2015). However, links among nutrient stoichiometry, eco-evolutionary feedbacks, and coevolution remain to be tested. 
Major advances in evolutionary ecology have been made through experimental studies of microbial communities. In particular, bacteria and phage are ideal for studying eco-evolutionary

94 feedbacks owing to their large population sizes, rapid growth rates, and experimental tractability. Moreover, bacteria and phage dynamics are critical for understanding the structure and function

96 of microbial food webs, especially in aquatic ecosystems (Suttle 2007). For example,

Synechococcus is a diverse and widely distributed group of primary producers in the world ocean with an estimated global abundance of $10^{26}$ cells (Flombaum et al. 2013). While Synechococcus must contend with both $\mathrm{N}$ - and P-limitation, it is also subject to a high degree of phage-induced mortality, which may lead to nutrient-dependent coevolutionary dynamics. These coevolutionary processes can create feedbacks that not only influence population dynamics, but also ecosystem 102 functioning, including the turnover of $\mathrm{N}$ and $\mathrm{P}$ (Lennon \& Martiny 2008).

In this study, we tested how nutrient stoichiometry affects host-parasite eco-evolutionary 104 dynamics in a simplified community where a single genotype of marine Synechococcus was infected with a phage strain in continuous culture using chemostats that were supplied with either $106 \mathrm{~N}$ - or P-limited media. We isolated hundreds of host and phage strains, which were challenged against one another to document phenotypic changes in resistance and infectivity over the course 108 of the experiment. With this information, we conducted network analyses that allowed us to quantify the degree of nestedness and modularity, which can serve as signatures of shed light on

110 the underlying modes of coevolution, namely arms race dynamics and negative frequency dependent selection (Weitz et al. 2013). Our findings reveal that nutrient stoichiometry is a

112 bottom-up force that regulates eco-evolutionary feedbacks in ways that alter coevolutionary processes. 


\section{METHODS}

116 Strains and media - We evaluated the effects of nutrient stoichiometry on the ecoevolutionary dynamics of the marine cyanobacterium Synechococcus WH7803 and a lytic T4-

118 like phage belonging to the Myoviridae family of phage (S-RIM8). Changes to nutrient supply can alter the equilibrium density of microbial populations in continuous culture (i.e.,

120 chemostats), which in turn, may affect cyanobacteria-phage contact rates (Rhee 1978). Therefore, we adjusted both the concentrations and ratios of $\mathrm{N}$ and $\mathrm{P}$ in modified $\mathrm{AN}$ artificial

122 seawater medium (Tables S1-S3) to induce N- or P-limitation while maintaining similar equilibrium densities of Synechococcus between the stoichiometry treatments prior to phage

124 addition. Specifically, the N-limited medium had an $\mathrm{N}: \mathrm{P}$ ratio of $10: 1\left(\mathrm{KNO}_{3} ; \mathrm{N}=220 \mu \mathrm{M}\right)$ while the P-limited medium had a $\mathrm{N}: \mathrm{P}$ ratio of $40: 1\left(\mathrm{~K}_{2} \mathrm{HPO}_{4} ; \mathrm{P}=11 \mu \mathrm{M}\right)$. Growth assays

126 confirmed that Synechococcus was limited by N under low N : P supply and by P under high N : P supply (Fig. S1).

Nutrient limitation assay — We tested for nutrient limitation in Synechococcus by measuring

130 growth rates on cells that had been maintained under low $(10: 1)$ and high $(40: 1)$ supply ratio of $\mathrm{N}$ and P. We acclimated Synechococcus semi-continuously under constant light $\left(20 \mu \mathrm{E} \mathrm{m}^{-1} \mathrm{~s}^{-1}\right)$

132 at $25^{\circ} \mathrm{C}$ for three serial transfers in either $\mathrm{N}$ - or P-limited medium $(\mathrm{n}=10)$. We then inoculated each replicate cell line into fresh medium that doubled the concentration of $\mathrm{N}(\mathrm{N}-$ limited $=440$

$134 \mu \mathrm{M}, \mathrm{P}$-limited $=880 \mu \mathrm{M})$ or $\mathrm{P}(\mathrm{N}$-limited $=44 \mu \mathrm{M}$, P-limited $=22 \mu \mathrm{M})$. Next, we monitored Synechococcus population densities for seven days via autofluorescence (ex: $550 \mathrm{~nm}$, em: 570

$136 \mathrm{~nm}$ ) with a Biotek Synergy Mx plate reader (Winooski, VT, USA). With these data, we estimated the maximum growth rate for each culture using a modified Gompertz equation 
138 (Lennon et al. 2007) and calculated the percent change in growth rate with additional $\mathrm{N}$ or $\mathrm{P}$ as compared to the control, which contained no additional $\mathrm{N}$ or $\mathrm{P}$.

Chemostat experiment - We supplied ten chemostats, each with a $40 \mathrm{~mL}$ operating volume,

142 with N-limited or P-limited medium at a dilution rate of $1 \mathrm{~d}^{-1}$ (Tables S1-S3). The chemostats were maintained in a Percival growth chamber (Perry, IA, USA) at $25^{\circ} \mathrm{C}$ on a 14:10 light : dark

144 cycle under $20 \mu \mathrm{E} \mathrm{m}^{-2} \mathrm{~s}^{-1}$ and homogenized with magnetic stir bars following inoculation with a single-colony strain of Synechococcus WH7803. We allowed the Synechococcus populations to

146 equilibrate in the chemostats prior to initiating the "phage-amended" treatment by introducing an aliquot of a plaque-purified S-RIM8 to three randomly chosen chemostats in each nutrient

148 treatment to achieve a multiplicity of infection (phage : cyanobacteria ratio) of approximately 10. To document the potential influence of stoichiometry on population dynamics and population

150 size, we maintained "no phage" chemostats with only Synechococcus in both stoichiometry treatments (Fig. S2).

Community dynamics - We tracked Synechococcus and phage densities in each chemostat via 154 epifluorescent microscopy every other day for 172 days. Synechococcus populations were enumerated by concentrating samples from a chemostat onto $0.22-\mu \mathrm{m}$ nominal pore-size black

156 polycarbonate filters. After removing cellular material $(0.2 \mu \mathrm{m}$ filtration $)$ and extracellular DNA with DNase I, we concentrated samples onto $0.02 \mu \mathrm{m}$ Anodisc filters to estimate the abundance

158 of free phage particles. Each phage-containing filter was then stained with SYBR Green I (Noble \& Fuhrman 1998). We estimated population densities after capturing ten images from 160 each filter with a CY3 filter set (ex: $550 \mathrm{~nm}$, em: $570 \mathrm{~nm}$ ) for Synechococcus or a FITC filter set 
(ex: $497 \mathrm{~nm}$, em: $520 \mathrm{~nm}$ ) for phage using a Zeiss microscope and image processing modules

162 found in the base package of the Axiovision imaging software (Release 4.5 SP1).

We evaluated the influence of nutrient stoichiometry on community dynamics in three

164 ways. First, we used repeated measures (RM) ANOVA to test for the effects of nutrient stoichiometry and time on Synechococcus and phage densities. Second, because cryptic

166 dynamics can alter the temporal synchrony between hosts and parasites, we performed crosscorrelation analyses for each chemostat using Auto Regressive Moving Average (ARMA)

168 procedures (Lennon \& Martiny 2008). In addition to determining whether populations in a chemostat positively or negatively covaried (i.e., in phase vs. out of phase, respectively) we used

170 the cross-correlation analyses to test if the duration of time which viruses lagged behind host densities was altered by nutrient stoichiometry. Last, we estimated the effects of nutrient

172 stoichiometry on the stability of microbial populations by calculating the inverse of the coefficient of variation (CV) over time.

Coevolutionary dynamics — To test for the effects of nutrient stoichiometry on phenotypic

176 coevolution, we tracked changes in infection patterns between Synechococcus and its phage over time. We isolated multiple (3-5) Synechococcus strains from each chemostat using serial dilution 178 techniques six days prior to phage addition (day -6) and at days 9, 23, 72, 129, 148, and 166 after phage addition. We performed 10-fold dilutions of chemostat samples into 24-well plates

180 containing sterile AN media that matched the nutrient supply ratio of the chemostat environment. When a dilute suspension of Synechococcus 7803 was incubated without shaking, single-

182 colonies formed in the bottom of the wells $\left(25^{\circ} \mathrm{C}\right.$ on a $14: 10$ light : dark cycle under $20 \mu \mathrm{E} \mathrm{m} \mathrm{m}^{-2} \mathrm{~s}^{-}$ $\left.{ }^{1}\right)$. We picked these colonies with a Pasteur pipette and passaged them twice in $50 \mathrm{~mL}$ 
184 Erlenmeyer flasks using the same medium prior to harvesting. We then used epifluorescence microscopy to screen the cultures to make sure they did not contain free phage particles. Finally, 186 these Synechococcus strains were concentrated via centrifugation, preserved in glycerol (10\% final concentration), and stored at $-80^{\circ} \mathrm{C}$ until reanimation for use in challenge assays, which are

188 described below. We also isolated multiple (3-5) phage strains from the phage-amended chemostats on days $23,72,129,148$, and 166 through double plaque purification. This process

190 involved adding $40 \mu \mathrm{L}$ of $0.2 \mu \mathrm{m}$-filtered chemostat sample to a soft agar ( $0.8 \%$ agar) overlay containing the ancestral Synechococcus WH7803 as the host. We then incubated the serially

192 diluted plates $\left(25^{\circ} \mathrm{C}\right.$ on a $14: 10$ light : dark cycle under $\left.20 \mu \mathrm{E} \mathrm{m}^{-2} \mathrm{~s}^{-1}\right)$, picked plaques that formed on the lawns of Synechococcus, and transferred to a log-phase batch culture of

194 Synechococcus for propagation. The resulting phage lysates were syringe-filtered $(1 \mu \mathrm{m})$ and preserved in glycerol (10\% final concentration) at $-80{ }^{\circ} \mathrm{C}$.

With the isolated chemostat strains, we quantified host resistance and phage infectivity using challenge assays. Each pair-wise challenge with strains isolated across chemostats within 198 the same nutrient treatment was carried out in triplicate by adding $20 \mu \mathrm{L}$ of a phage stock $\left(\sim 10^{7}\right.$ particles $\left.\mathrm{mL}^{-1}\right)$ to $200 \mu \mathrm{L}$ of a dilute Synechococcus strain $\left(\sim 10^{6}\right.$ cells $\left.\mathrm{mL}^{-1}\right)$ in $96-\mathrm{well}$ plates.

200 Challenge assays were performed using the same medium from which the host strain was originally isolated (i.e., low vs. high N : P). Turbid cultures of Synechococcus WH7803 appear

202 bright pink owing to the intracellular photosynthetic pigment phycoerythrin. When infected by SRIM8, however, cells are lysed and cultures become clear (Lennon \& Martiny 2008). Based on 204 this, we scored each Synechococcus strain as sensitive (and the phage strain as infective) if there was a lack of growth after a two-week incubation under continuous light $\left(20 \mu \mathrm{E} \mathrm{m} \mathrm{m}^{-2} \mathrm{~s}^{-1}\right)$ at $25^{\circ} \mathrm{C}$ 206 compared to control wells $(n=3)$ that contained heat-killed phage. To contend with the loss of a 
replicate chemostat in the N-limited treatment, we randomly selected Synechococcus strains across the two P-limited replicate chemostats to ensure that an identical number of strains were tested at each time point. In total, there were 18,050 pairwise challenges between chemostat-

210 isolated strains of Synechococcus and phage that resulted in a bipartite infection matrix, which we used for all analyses related to phenotypic coevolution.

To quantify the effect of nutrient stoichiometry on coevolutionary dynamics, first, we used RM-ANOVA to test for trends in average resistance and average infectivity over the course

214 of the chemostat experiment. Second, we used the infection matrix to perform time-shift analyses, which involved calculating the proportion of successful infections that occurred

216 between hosts that were challenged against past, contemporary, and future phage strains (Gaba \& Ebert 2009). We statistically analyzed the time-shift data using RM-ANOVA with nutrient

218 treatment, phage treatment, and time as fixed effects, while the chemostat replicate identifier was treated as a random effect with a corARMA covariance matrix (Koskella 2014). To visualize the 220 data, contemporary challenges were centered at a time-shift of zero, while interactions with past phage were represented in negative space and future interactions were represented in positive

222 space. Last, to gain insight into potential mechanisms underlying stoichiometrically driven coevolution, we used community network analyses to calculate the connectance, nestedness, and 224 modularity for each chemostat infection matrix. Connectance was calculated as the number of interactions divided by network size. We calculated nestedness using the NODF metric, which 226 ranges from 0 (non-nested) to 1 (perfectly nested) and normalizes for matrix size. We used the LP-BRIM algorithm to find the partition that maximizes Barber's modularity $(\mathrm{Qb})$, which ranges 228 from 0 (all interactions are between modules) to 1 (all interactions are within modules). The network statistics were calculated using 100,000 random Bernoulli simulations in the BiWeb 
230 package for MATLAB (Flores et al. 2011; Flores et al. 2016), http://github.com/tpoisot/BiWeb). We then tested for differences in connectance, nestedness, and modularity between the nutrient 232 treatments using $t$-tests.

\section{RESULTS}

Nutrient limitation of Synechococcus - Prior to initiating the chemostat evolution trial, we

236 conducted growth assays to test for N- and P-limitation of Synechococcus. After a period of acclimation in semi-continuous culture, the growth rates of N-limited Synechococcus increased

238 by $87 \%$ when additional $\mathrm{N}$ was supplied, but only by $35 \%$ with the addition of $\mathrm{P}\left(t\right.$-test, $t_{8}=$ 4.55, $P<0.001)$. In contrast, the growth rate of P-limited Synechococcus increased by $45 \%$ with

240 the addition of $\mathrm{P}$, but only by $25 \%$ with the addition of $\mathrm{N}\left(t\right.$-test, $\left.t_{8}=-3.36, P=0.005\right)$. Results from this experiment suggest that while there may have been some degree of co-limitation, the

242 two media sources used for subsequent evolution trials induced N- and P- limitation for Synechococcus (Fig. S1).

Stoichiometry altered community dynamics — In the chemostat experiment, nutrient

246 stoichiometry significantly affected the population dynamics of Synechococcus (RM-ANOVA; time x stoichiometry, $\left.F_{62,245}=2.43, P<0.0001\right)$ and its phage (RM-ANOVA; time x

248 stoichiometry, $\left.F_{58,230}=2.59, P<0.0001\right)$. Under N-limitation, Synechococcus rapidly declined and reached its minimum mean density 35 days (range $=23-44)$ following phage addition $(2 \mathrm{x}$ $25010^{5} \pm 7 \times 10^{4}$ cells $\mathrm{mL}^{-1}$, mean \pm SEM, Fig. 1a). This decrease in host abundance corresponded with a peak in mean maximum density 25 days (range $=16-30)$ following phage addition $(9 \mathrm{x}$ 
$25210^{8} \pm 1 \times 10^{8}$ particles $\mathrm{mL}^{-1}$, mean \pm SEM). Over the next $\sim 50$ days, the host densities slowly recovered, and entered a second phase of decline near day 100.

Synechococcus and phage densities were significantly different and less dynamic under P-limitation (Fig. 1b). Hosts declined more slowly in P-limited chemostats following phage

256 addition than in $\mathrm{N}$-limited chemostats $\left(t\right.$-test, $\left.t_{4}=-4.00, P=0.02\right)$ and reached their mean minimum density $78($ range $=63-93)$ days following phage addition $\left(4 \times 10^{5} \pm 6 \times 10^{4}\right.$ cells

$258 \mathrm{~mL}^{-1}$, mean $\left.\pm \mathrm{SEM}\right)$. Though hosts recovered, they did not reach pre-phage-addition abundances until the end of the experiment. Phage densities under P-limitation remained high and relatively

260 constant $\left(2 \times 10^{8} \pm 9 \times 10^{7}\right.$ particles $\mathrm{mL}^{-1}$, mean $\left.\pm \mathrm{SEM}\right)$ throughout the duration of the experiment. In the no-phage control chemostats, N- and P- limited Synechococcus densities

262 remained constant over time (Fig. S2).

Nutrient stoichiometry also affected the temporal coherence and stability of

264 Synechococcus and phage populations. After pre-whitening the time-series data using ARMA procedures, phage densities in N-limited chemostats were negatively correlated with host

266 densities with lags ranging from zero to five days ( $r=-0.27$ to $-0.42, P=0.009-0.063$, Fig. S3). In contrast, under P-limitation, there was no correlation between phage and Synechococcus at 268 any time lag $(r=-0.20$ to $0.22, P \geq 0.23)$. Last, we found that cyanobacterial and phage densities were significantly more stable over time under P- than N-limited conditions (Synechococcus: $t$ 270 test, $t_{4}=3.56, P=0.024$; $t$-test, phage: $\left.t_{4}=3.83, P=0.019\right)$. See Table $\mathrm{S} 4$ for summary statistics.

272 Stoichiometry altered coevolution - Within nine days of phage addition, $>50 \%$ of the isolated Synechococcus strains were resistant to the ancestral phage in both the N- and P-limited 274 chemostats (Fig. 2). Average resistance continued to increase over time (RM-ANOVA, $F_{6,591}=$ 
14.2, $P<0.0001)$, but was not affected by stoichiometry $\left(F_{1,4}=2.08, P=0.22\right)$. The vast majority (97\%) of Synechococcus strains that were resistant to the ancestral phage were also resistant to all of the phage strains subsequently isolated from the chemostats. Phage-resistant 278 Synechococcus did not revert to the sensitive phenotype when grown in the absence of phage consistent with the view that resistance is a heritable trait. nutrient stoichiometry (Fig. 2; RM-ANOVA, stoichiometry x time: $F_{1,601}=7.53, P=0.006$ ). In

282 the no-phage control chemostats, Synechococcus strains remained susceptible to the ancestral phage. However, nearly all $(\sim 96 \%)$ of the derived phage from the phage-amended chemostats

284 lost the ability to infect some of the Synechococcus hosts from the P-limited $(\mathrm{n}=51)$ and Nlimited $(n=22)$ no-phage chemostats. Despite this, we found evidence of host-range expansion.

286 For example, a phage strain from a P-limited chemostat (day 129) was able to infect a Synechococcus strain (day 166) that was resistant to the ancestral phage. In addition, three phage strains from N-limited chemostats and two phage strains from P-limited chemostats (day 166) were able to infect phage-resistant Synechococcus isolated from other chemostats earlier in the 290 study.

Time-shift experiments indicated that nutrient stoichiometry altered coevolutionary

292 dynamics (Figs. 3 and 4). When hosts isolated from the phage-amended chemostats were challenged against current and past phage, interaction strengths were generally weak owing to

294 the evolution of host resistance and phage infectivity (Fig. 3 and Fig 4 a, b; RM-ANOVA, stoichiometry x phage isolation time $\mathrm{x}$ bacterial isolation time, $\left.F_{1,597}=24.67, P<0.0001\right)$. Hosts

296 were more susceptible when challenged against future phage, but only for Synechococcus strains that were isolated earlier in the chemostat experiment (days $-6,9$, and 23). The effect of nutrient 
stoichiometry was more evident in time-shift experiments where naïve hosts from the no-phage control chemostats were challenged against phage isolated from the phage-amended chemostats

300 (stoichiometry x phage isolation time, $\mathrm{F}_{1,282}=7.25, P=0.0075$ ). Under these conditions, infectivity was not uniformly high across the time-shifts (Fig 3 and Fig. 4 c, d), as would be

302 expected from purely arms-race dynamics. As a result, infectivity was $70 \%$ greater on naïve hosts isolated from N-limited chemostats $(0.61 \pm 0.289)$ compared to naïve hosts isolated from

304 P-limited chemostats $(0.36 \pm 0.332)$.

Network analyses lent further support that nutrient stoichiometry affected host-phage coevolution. Patterns of infectivity were more nested and modular than expected by chance $(P<$ 0.05), which is consistent with arms race dynamics and negative frequency-dependent selection, respectively. The infection networks from $\mathrm{N}$ - and P-limited environments had similar degrees of connectance $\left(t_{4}=1.47, P=0.22\right)$ and nestedness (NODF, $\left.t_{4}=-0.37, P=0.72\right)$. However, P-

310 limited networks were $50 \%$ more modular than N-limited networks (Fig. $5 ; t_{4}=-3.59, P=0.02$ ). See Table S6 for summary statistics.

\section{DISCUSSION}

314 The supply ratio of nitrogen $(\mathrm{N})$ and phosphorus $(\mathrm{P})$ had strong eco-evolutionary effects on marine Synechococcus and its phage. Communities were more stable under P-limitation than

316 N-limitation, which likely was influenced by the nutritional flexibility and constraints of the host and phage populations, respectively. In addition, host-phage dynamics were influenced by the

318 rapid invasion of resistant cyanobacteria which was followed by the appearance of evolved phage with altered host-ranges. Using time-shift assays and network analyses, we detected strong 320 signatures of arms-race dynamics in both nutrient treatments. However, under P-limitation, we 
also observed patterns of increased network modularity consistent with negative frequency-

322 dependent selection, which may reflect the underlying costs of maintaining defense and virulence traits in different nutrient environments. Our findings generate testable predictions

324 regarding the mechanisms of local adaptation and coevolution for microorganisms among regions of the world ocean that are limited to varying degrees by $\mathrm{N}$ and $\mathrm{P}$ (Galbraith \& Martiny 326 2015).

328 Stoichiometry altered community dynamics - Consistent with predictions from the theory of ecological stoichiometry (Sterner \& Elser 2002), we documented that Synechococcus and phage

330 dynamics were highly sensitive to the $\mathrm{N}$ : P supply ratio. Under N-limitation, host-phage dynamics were less stable and out-of-phase, compared to P-limited chemostats (Fig. 1; Table

332 S4). Historically, ecologists have attempted to explain such variation in populations and communities while assuming a single currency of resource. However, systems can exhibit a much wider range of behaviors when resource stoichiometry is explicitly considered owing to feedbacks that arise when interacting species vary in the degree to which their elemental 336 composition is homeoestatically regulated (Andersen et al. 2004). For example, the molar $\mathrm{N}: \mathrm{P}$ ratio of marine Synechococcus converges upon the canonical Redfield value of $16: 1$ in some

338 environments (Garcia et al. 2016), but in other instances can exceed $100: 1$ (Bertilsson et al. 2003) reflecting the extreme plasticity of its biomass stoichiometry. In contrast, phage are

340 thought to have a fixed elemental composition owing to their relatively simple structure, which minimally consists of genetic material (DNA or RNA) protected by a proteinaceous capsid

342 (Jover et al. 2014). Biophysical models predict that the T4-like phage used in our study has a high P demand reflected by its low $\mathrm{N}$ : P ratio of approximately $7: 1$ (Jover et al. 2014). As a 344 consequence, phage productivity is largely dependent on the P content of their hosts. For 
example, host lysis was delayed by $18 \mathrm{~h}$ leading to an $80 \%$ reduction in phage burst size when with these reports, early in our experiment (day -6), phage infectivity was $50 \%$ lower on P-

348 limited vs. N-limited hosts (cf. Fig. 4A and 4B). It is possible, however, for phage to overcome the nutritional constraints of their microbial hosts. Many phage contain auxiliary metabolic genes that modify a host's resource allocation (Monier et al. 2017). For example, phage-encoded genes for phosphorus acquisition ( $p s t S$ and $p h o A$ ) were upregulated when phosphorus-starved marine

352 Prochlorococcus was infected by phage P-SSM2 (Zeng \& Chisholm 2012). While the myovirus used in our study (S-RIM8) does not contain phosphate-binding protein or alkaline phosphatase

354 genes, it does contain $p h o H$. The exact function of $p h o H$ in marine microorganisms has not been demonstrated, but it is thought to encode a phosphate-starvation inducible protein that

356 hydrolyzes ATP, thus liberating energy that can be used in P uptake (Goldsmith et al. 2011). Such findings suggest that nutrient stoichiometry can profoundly shape host-parasite dynamics

358 (Aalto et al. 2015) and provide an ecological explanation for the patterns observed in our study (Fig. 1).

Stoichiometry altered coevolution — Our results indicate that evolutionary processes were also 362 important in creating feedbacks that influenced host-phage dynamics. Such feedbacks arise when rapid changes in host or parasite traits generate patterns that are not predicted from traditional 364 ecological theory (Yoshida et al. 2007; Frickel et al. 2016). These so-called cryptic dynamics require the coexistence of multiple host genotypes, for example, through trade-offs in

366 competitive ability and parasite defense (Yoshida et al. 2007). While there are many intracellular and extracellular mechanisms that bacteria can employ to resist phage infection, in 
368 Synechococcus, mutations in genes encoding for cell-surface receptors reduce or entirely eliminate attachment, thus precluding entry of the virus into the host (Stoddard et al. 2007;

370 Marston et al. 2012). Selection for this phenotype could help explain the recovery of host densities in our chemostats (Fig. 1). However, resistance mutations in Synechococcus are often

372 accompanied by a reduction in growth rate, the magnitude of which can vary depending on the identity of the host and virus (Lennon 2007). These fitness costs have important consequences

374 for understanding community stability. Without a reduction in growth rate, resistant Synechococcus would outcompete the sensitive host and drive the phage population extinct.

376 Moreover, fitness costs establish a trade-off that satisfies the requirement for cryptic dynamics to emerge (Yoshida et al. 2007). In sum, our data support the view that nutrient stoichiometry

378 altered host-phage dynamics and stability via eco-evolutionary feedbacks (Lennon \& Martiny 2008).

Nutrient stoichiometry had strong, yet importantly, asymmetric effects on microbial coevolution in our study. These effects were examined in light of the two primary modes by 382 which antagonistic coevolution is thought to occur. The first is through arms-race dynamics involving gene-for-gene specificity, where directional selection leads to sweeps that are

384 characterized by the escalation of resistance and infectivity. Arms-race dynamics were originally described for coevolving populations of plants and pathogens, but since then have been

386 commonly reported in studies of bacteria and phage (Dennehy 2012; Koskella \& Brockhurst 2014). The second mode of antagonistic coevolution involves negative frequency-dependent 388 selection where parasites evolve to infect common hosts, which in turn favors rare host alleles. Negative frequency-dependent selection is often associated with infections that require matching 390 alleles and is well documented in invertebrate systems (Decaestecker et al. 2007), but has also 
been described in some studies of bacteria and phage (Hall et al. 2011). While arms-race dynamics and negative frequency-dependent selection are often viewed as occupying different ends of the coevolutionary spectrum, they are not mutually exclusive (Agrawal \& Lively 2002). One powerful way to discern modes of coevolution is through the use of time-shift analyses. This approach involves determining the success of infections for combinations of hosts and parasites that are isolated from different time points in a controlled experiment or other longitudinal type of study (Gaba \& Ebert 2009). When applied to challenge assays among host and phage isolates from our chemostats, time-shift analyses indicated that coevolutionary dynamics were significantly affected by nutrient stoichiometry (Fig. 3). The effect of nutrient stoichiometry was relatively weak when examining host strains isolated from phage-amended chemostats, which could reflect a mismatch between the time-shift interval and the rates of 402 coevolution (Gandon et al. 2008). In contrast, the effect of nutrient stoichiometry was driven largely by interactions between evolved viruses that were challenged against Synechococcus

404 isolated from no-phage control chemostats (Fig. 4 c, d). We expected that these naïve hosts would be uniformly and highly susceptible to all phage from the phage-amended chemostats.

406 Instead, we found that infectivity fluctuated over time and was higher on N-limited vs. P-limited Synechococcus, reflecting expansion and contraction of the phage host-range (Fig. 4 c, d).

408 Together, these findings are consistent with observations of asymmetric coevolution that have been attributed to the genetic constraints of viral populations (Lenski \& Levin 1985). Asymmetry 410 may also arise from the costs associated with being a generalist phage (Koskella \& Brockhurst 2014), which may be dependent on the resource environment (e.g., nutrient stoichiometry). Such

412 explanations have been used to explain host-phage coevolution where arms race dynamics give 
way to negative frequency dependent selection (Hall et al. 2011), in some cases depending on

414 resource availability (Pascua et al. 2014).

The effects of nutrient stoichiometry on coevolution were further supported by results

416 from our network analyses. With this approach, one can estimate the degree of nestedness that exists among pairs of hosts and phage relative to randomized data. Often, infection data from

418 bacteria-phage systems are highly nested (Flores et al. 2011), which means they contain ordered subsets of phenotypes whereby hosts from later time points are resistant to earlier phages, and 420 phage from later time points are able to infect earlier hosts (Weitz et al. 2013). In our study, infection networks were significantly nested, a pattern that arises from arms-race dynamics.

422 However, the degree of nestedness was not affected by nutrient stoichiometry. Infection networks can also exhibit modularity, which is more consistent with negative frequency-

424 dependent selection (Weitz et al. 2013). Modules reflect dense clusters of interacting host and phage compared to other strain combinations found in a bipartite infection matrix. Although less 426 commonly documented, modularity has been observed in large-scale oceanic surveys of bacteria and phage, but has been attributed to local adaptation and the phylogenetic breadth of the hosts

428 (Flores et al. 2013). However, theory suggests that modularity can emerge via coevolution between two populations at the local scale. Using a "relaxed lock and key" model,

430 simultaneously nested and modular structures arose under simple chemostat conditions that assumed gene matching between phage tail-fibers and host receptors (Beckett \& Williams 2013).

432 Our results agree with these predictions, but suggest that network properties are affected by host nutrition. Specifically, we found that host-phage interactions were $50 \%$ more modular under P-

434 limitation than N-limitation, suggesting that nutrient stoichiometry constrains host-phage interactions leading to increased specialization. Such findings are consistent with the view that 
436 resources can influence the modes of coevolution based in part on the fitness costs associated with host defense and infection strategies (Pascua et al. 2014) Mechanistically, there are many ways that nutrient stoichiometry could shape bacteriaphage coevolution. For example, the "dangerous nutrients" hypothesis predicts that trajectories 440 of coevolution can be influenced when receptors used by nutrient-limited hosts also serve as the targets of phage adsorption (Menge \& Weitz 2009). However, it does not appear that

442 myoviruses, including the strain used in this study, attach to the protein receptors of cyanobacteria that are used for nutrient transport. Instead, evidence from whole-genome

444 sequencing of the host used in our study suggests that phage-resistant Synechococcus accumulate mutations in hypervariable genomic islands that encode for lipopolysaccharide (LPS) (Marston 446 et al. 2012), a major component of the outer membrane in Gram-negative bacteria. The structural complexity of LPS is affected by nutrient limitation (Brelles-Marino \& Boiardi 1997) and such

448 changes in molecular structure of the cell membrane can interfere with phage adsorption leading to resistance (Leon \& Bastias 2015). Nevertheless, modification of tail-fibers allow phage to 450 overcome resistance of marine cyanobacteria in some instances (Schwartz \& Lindell 2017) while the acquisition of host-like P-assimilation genes may aid in the successful infection of nutrient-

452 limited hosts (Kelly et al. 2013).

454 Conclusions - Most organisms live in environments where they are limited by the relative or absolute amount of one or more essential resources. It is well established that such variation in 456 nutrient stoichiometry can regulate ecological phenomena ranging from species interactions to ecosystem-level processes. We demonstrated that nutrient stoichiometry also affects the 458 evolutionary dynamics of microbial communities likely through differences in the expansions 
and contraction of virus host-ranges under N- vs. P-limited conditions. Identifying the targets of selection in contrasting nutrient environments will help elucidate the genetic mechanisms of coevolution and the trade-offs associated with defense and virulence traits. While our study

462 offers promising avenues to better understand bacteria-phage interactions in the aquatic environments, nutrient stoichiometry is also important in determining the nutrition, health, and

464 disease susceptibility for non-microbial hosts and their microbiomes(Smith \& Holt 1996), which has implications for understanding the persistence, emergence, and evolution of infectious

466 diseases. In the oceans, phage represent a significant source of mortality for marine cyanobacteria like Synechococcus, which play a central role in the regulation of biogeochemical 468 processes, including energy flow, carbon sequestration, and the cycling of nitrogen (N) and phosphorus (P). Our findings suggest that the coevolutionary process was differentially affected

470 by the availability of $\mathrm{N}$ and $\mathrm{P}$ in ways that could influence the ecology and evolution of one of the most abundant and functionally important groups of microorganisms on Earth.

\section{Acknowledgments}

474 We acknowledge technical support from R Morrison, M Carroll, and BK Lehmkuhl; ancestral strains from MF Marston; discussion with CM Lively; feedback from BK Whitaker, KJ Locey,

476 WR Shoemaker, NI Wisnoski, V Kuo, ME Muscarella, DA Schwartz, RZ Moger-Reischer, JM Palange on earlier versions of the manuscript. Financial support was provided by the National

478 Science Foundation (0851143, 0851113) and the US Army Research Office Grant W911NF-141-0411. We dedicate this work to the memory of VH Smith. 


\section{REFERENCES}

1.

484 Aalto, S.L., Decaestecker, E. \& Pulkkinen, K. (2015). A three-way perspective of stoichiometric changes on host-parasite interactions. Trends Parasitol., 31, 333-340.

4862.

Acquisti, C., Elser, J.J. \& Kumar, S. (2009). Ecological Nitrogen Limitation Shapes the DNA Composition of Plant Genomes. Mol. Biol. Evol., 26, 953-956.

3.

490 Agrawal, A. \& Lively, C.M. (2002). Infection genetics: gene-for-gene versus matching-alleles models and all points in between. Evol. Ecol. Res., 4, 79-90.

4924.

Andersen, T., Elser, J.J. \& Hessen, D.O. (2004). Stoichiometry and population dynamics. Ecol. Lett., 7, 884-900.

5.

496 Beckett, S.J. \& Williams, H.T.P. (2013). Coevolutionary diversification creates nested-modular structure in phage-bacteria interaction networks. Interface Focus, 3: 20130033.

4986.

Bertilsson, S., Berglund, O., Karl, D.M. \& Chisholm, S.W. (2003). Elemental composition of marine Prochlorococcus and Synechococcus: Implications for the ecological stoichiometry of the sea. Limnol Oceanogr, 48, 1721-1731.

7.

Bragg, J.G. \& Wagner, A. (2009). Protein material costs: single atoms can make an evolutionary difference. Trends Genet., 25, 5-8.

8.

506 Brelles-Marino, G. \& Boiardi, J.L. (1997). $\mathrm{Mn}^{2+}$ deficiency affects growth energetics of Rhizobium etli. Microbiol. Res., 152, 367-371.

5089.

Decaestecker, E., Gaba, S., Raeymaekers, J.A.M., Stoks, R., Van Kerckhoven, L., Ebert, D. et al. (2007). Host-parasite 'Red Queen' dynamics archived in pond sediment. Nature, 450, 870U816.

10.

Dennehy, J.J. (2012). What can phages tell us about host-pathogen coevolution? Int. J. Evol. Biol.,

11.

516 Elser, J.J., Acquisti, C. \& Kumar, S. (2011). Stoichiogenomics: the evolutionary ecology of macromolecular elemental composition. Trends Ecol. Evol., 26, 38-44.

51812.

Flombaum, P., Gallegos, J.L., Gordillo, R.A., Rincon, J., Zabala, L.L., Jiao, N.A.Z. et al. (2013). Synechococcus. Proc. Natl Acad. Sci. U S A, 110, 9824-9829.

13.

Flores, C.O., Meyer, J.R., Valverde, S., Farr, L. \& Weitz, J.S. (2011). Statistical structure of hostphage interactions. Proc. Natl Acad. Sci. US A, 108, E288-E297.

14. 
526 Flores, C.O., Poisot, T., Valverde, S. \& Weitz, J.S. (2016). BiMat: a MATLAB package to facilitate the analysis of bipartite networks. Methods Ecol. Evol., 7, 127-132.

15.

Flores, C.O., Valverde, S. \& Weitz, J.S. (2013). Multi-scale structure and geographic drivers of cross-infection within marine bacteria and phages. ISME J., 7, 520-532.

16. Frickel, J., Sieber, M. \& Becks, L. (2016). Eco-evolutionary dynamics in a coevolving hostvirus system. Ecol. Lett., 19, 450-459.

17.

536 Gaba, S. \& Ebert, D. (2009). Time-shift experiments as a tool to study antagonistic coevolution. Trends Ecol. Evol., 24, 226-232.

18.

Galbraith, E.D. \& Martiny, A.C. (2015). A simple nutrient-dependence mechanism for predicting

19. the stoichiometry of marine ecosystems. Proc. Natl Acad. Sci. U S A, 112, 8199-8204.

542 Gandon, S., Buckling, A., Decaestecker, E. \& Days, T. (2008). Host-parasite coevolution and patterns of adaptation across time and space. J. Evol. Biol. 21, 1861-1866.

20.

546 Garcia, N.S., Bonachela, J.A. \& Martiny, A.C. (2016). Interactions between growth-dependent changes in cell size, nutrient supply and cellular elemental stoichiometry of marine Synechococcus. ISME J., 10, 2715-2724.

21.

550 Goldsmith, D.B., Crosti, G., Dwivedi, B., McDaniel, L.D., Varsani, A., Suttle, C.A. et al. (2011). Development of $\mathrm{phoH}$ as a novel signature gene for assessing marine phage diversity. Appl Environ Microbiol, 77, 7730-7739.

22.

554 Gresham, D., Desai, M.M., Tucker, C.M., Jenq, H.T., Pai, D.A., Ward, A. et al. (2008). The repertoire and dynamics of evolutionary adaptations to controlled nutrient-limited

23. environments in yeast. PLoS Genet., 4.

558 Hall, A.R., Scanlan, P.D., Morgan, A.D. \& Buckling, A. (2011). Host-parasite coevolutionary arms races give way to fluctuating selection. Ecol. Lett., 14, 635-642.

56024.

Hall, S.R. (2009). Stoichiometrically explicit food webs: feedbacks between resource supply,

562 elemental constraints, and species diversity. Annu. Rev. Ecol. Evol. Syst., 40. 503-528. 25.

564 Haloin, J.R. \& Strauss, S.Y. (2008). Interplay between ecological communities and evolution. Ann. N.Y. Acad. Sci., 1133, 87-125.

56626.

Jover, L.F., Effler, T.C., Buchan, A., Wilhelm, S.W. \& Weitz, J.S. (2014). The elemental 568 composition of virus particles: implications for marine biogeochemical cycles. Nat. Rev. Microbiol., 12, 519-528. 
Kelly, L., Ding, H.M., Huang, K.H., Osburne, M.S. \& Chisholm, S.W. (2013). Genetic diversity in cultured and wild marine cyanomyoviruses reveals phosphorus stress as a strong selective agent. ISME J., 7, 1827-1841.

57428.

Kimura, M. (1962). On the probability of fixation of mutant genes in a population. Genetics, 47 , 713-719.

29.

578

580

582

584

586

588

590

592

34.

31.

30.

Koskella, B. \& Brockhurst, M.A. (2014). Bacteria-phage coevolution as a driver of ecological and evolutionary processes in microbial communities. FEMS Microbiol. Rev., 38, 916-931.

Lennon, J.T. (2007). Is there a cost of viral resistance in marine cyanobacteria? ISME J., 1, 300312.

32.

Lennon, J.T. \& Martiny, J.B.H. (2008). Rapid evolution buffers ecosystem impacts of viruses in a microbial food web. Ecol. Lett., 11, 1178-1188.

33.

594 Leon, M. \& Bastias, R. (2015). Virulence reduction in bacteriophage resistant bacteria. Front. Microbiol., 6.

$596 \quad 35$

Marston, M.F., Pierciey, F.J., Shepard, A., Gearin, G., Qi, J., Yandava, C. et al. (2012). Rapid

598

600

36. diversification of coevolving marine Synechococcus and a virus. Proc. Natl Acad. Sci. US $A$, , 109, 4544-4549.

Menge, D.N.L. \& Weitz, J.S. (2009). Dangerous nutrients: Evolution of phytoplankton resource

602 37. uptake subject to virus attack. J Theor Bio, 257, 104-115.

604 Monier, A., Chambouvet, A., Milner, D.S., Attah, V., Terrado, R., Lovejoy, C. et al. (2017). Host-derived viral transporter protein for nitrogen uptake in infected marine

606 38. phytoplankton. Proc. Natl Acad. Sci. U S A, 114: E7489-E7498.

608 Noble, R.T. \& Fuhrman J.A. (1998). Use of SYBR Green 1 for rapid epiluorescence count of marine viruses and bacteria. Aquat. Microb. Ecol. 14, 113-118.

61039.

612

Pascua, L.L., Hall, A.R., Best, A., Morgan, A.D., Boots, M. \& Buckling, A. (2014). Higher

614 1380-1388.

40.

Rhee, G.Y. (1978). Effects of N:P atomic ratios and nitrate limitation on algal growth cell composition, and nitrate uptake. Limnol. Oceanogr., 23, 10-25. 
41.

618 Schwartz, D.A. \& Lindell, D. (2017). Genetic hurdles limit the arms race between Prochlorococcus and the T7-like podoviruses infecting them. ISME J., 11, 1836-1851.

42.

Smith, V.H. \& Holt, R.D. (1996). Resource competition and within-host disease dynamics. Trends Ecol. Evol., 11, 386-389.

43.

624 Sterner, R.W. \& Elser, J.J. (2002). Ecological Stoichiometry: The Biology of Elements from Molecules to the Biosphere. Princeton University Press.

62644

Stoddard, L.I., Martiny, J.B.H. \& Marston, M.F. (2007). Selection and characterization of cyanophage resistance in marine Synechococcus strains. Appl. Environ. Microbiol., 73, 5516-5522.

63045.

Suttle, C.A. (2007). Marine viruses - major players in the global ecosystem. Nat. Rev. Microbiol., $5,801-812$.

46.

634 Turner, C.B., Wade, B.D., Meyer, J.R., Sommerfeld, B.A. \& Lenski, R.E. (2017). Evolution of organismal stoichiomery in a long-term experiment with Escherichia coli. R. Soc. Open

47. Sci., DOI: 10.1098/rsos. 170497

638 Van Mooy, B.A.S., Rocap, G., Fredricks, H.F., Evans, C.T. \& Devol, A.H. (2006). Sulfolipids dramatically decrease phosphorus demand by picocyanobacteria in oligotrophic marine environments. Proc. Natl Acad. Sci. US A, 103, 8607-8612.

48.

642 Weitz, J.S., Poisot, T., Meyer, J.R., Flores, C.O., Valverde, S., Sullivan, M.B. et al. (2013). Phagebacteria infection networks. Trends Microbiol., 21, 82-91.

64449.

Wilson, W.H., Carr, N.G. \& Mann, N.H. (1996). The effect of phosphate status on the kinetics of 646 cyanophage infection in the oceanic cyanobacterium Synechococcus sp WH7803. J. Phycol., 32, 506-516.

64850.

Yamamichi, M., Meunier, C.L., Peace, A., Prater, C. \& Rua, M.A. (2015). Rapid evolution of a 650 consumer stoichiometric trait destabilizes consumer-producer dynamics. Oikos, 124, 960969.

65251

Yoshida, T., Ellner, S.P., Jones, L.E., Bohannan, B.J.M., Lenski, R.E. \& Hairston, N.G. (2007).

654 Cryptic population dynamics: Rapid evolution masks trophic interactions. PLoS. Biol., 5, 1868-1879.

65652.

Zeng, Q. \& Chisholm, S.W. (2012). Marine viruses exploit their host's two-component regulatory 658 system in response to resource limitation. Curr Biol, 24. 


\section{FIGURE CAPTIONS}

Fig. 1. Microbial community dynamics were affected by nutrient stoichiometry. Synechococcus

664 and phage densities were tracked in replicate $(n=3)$ chemostats receiving nitrogen $(\mathrm{N})$ - or phosphorus (P)-limited media. Vertical lines at day 0 indicate time of phage amendment. See

666 Fig. S2 for Synechococcus dynamics in the no-phage control chemostats. Data are represented as mean \pm SEM.

Fig. 2 Average phage-resistance of Synechococcus strains increased monotonically over time 670 under both nitrogen (N)- and phosphorus (P)-limitation (left). Resistance was calculated as the proportion of unsuccessful infections between all phage strains from the experiment when

672 challenged against Synechococcus strains that had been isolated at a specific time point and is presented as the chemostat mean \pm SEM. Average infectivity of phage over time (right) was calculated as the proportion of successful infections between all Synechococcus strains across the experiment when challenged against phage strains that had been isolated (on the ancestral host)

676 at a specific time point and is presented as the chemostat mean \pm SEM.

678 Fig. 3 Phenotypic coevolution between hosts (Synechococcus) and phage was affected by nutrient stoichiometry. We calculated infectivity based on the proportion of successful infections 680 between Synechococcus strains and phage strains that were isolated from chemostats at different time points. Infectivity is proportional to the width of the edges (lines) connecting nodes

682 (symbols). Black squares correspond to phage isolated from the phage-amended chemostats, white circles correspond to Synechococcus isolated from phage-amended chemostats, and grey 684 circles correspond to naive Synechococcus isolated from no-phage control chemostats. The 
absence of a line indicates that Synechococcus isolates were resistant to a particular phage challenge.

688 Fig. 4. Time-shift analysis of host-phage infectivity reveals the effects of stoichiometry on coevolution. Contemporary interactions (i.e., those between a host and phage strain isolated at

690 the same time point) are centered at time zero (grey vertical line) along the time-shift (horizontal) axis. Interactions with past phage are shifted to the left (negative values) and

692 interactions with future phage are shifted to the right (positive values). Each black line corresponds with the mean infectivity for Synechococcus isolated from a specific time point as

694 indicated by the open circle containing the isolation day $(-6,9,23,72,100,129$, or 166$)$. When comparing challenges between hosts and phage from phage-amended chemostats $(a, b)$,

696 infectivity was weak for hosts that were isolated after day 23 or when challenged against phage from the past owing to the evolution of resistance. Such findings are consistent with arms-race 698 dynamics where directional selection gives rise to escalating host resistance. We also challenged phage against naive hosts from the no-phage control chemostats $(c, d)$. From this, we found that

700 infectivity was significantly higher under nitrogen $(\mathrm{N})$-limitation than phosphorus (P)-limitation, but overall, was lower than what would be expected under arms-race dynamics. Instead, the 702 fluctuations in infectivity with respect to time-shift are consistent with negative frequencydependent selection and reflect asymmetry in the coevolution between Synechococcus and 704 phage.

706 Fig. 5. Host-phage infection networks based on interactions between Synechococcus and phage isolates that coevolved under nitrogen $(\mathrm{N})$ - and phosphorus $(\mathrm{P})$-limitation. Networks were 
708 significantly nested, consistent with expectations of host-phage systems coevolving under arms race dynamics. However, the degree of nestedness was not affect by stoichiometry. Left panel:

710 Networks were also significantly modular when compared to randomized networks, but the degree of modularity was significantly greater in P-limited networks. Right panel: Infection

712 networks reflecting the modular structure of host-phage interactions within each chemostat. Each grouping of colored cells within a grey-colored background panel corresponds to a calculated

714 module within the interaction network of N-limited $(a-c)$ or P-limited $(d-f)$ chemostats. That is, colored cells correspond to infectious interactions that could not be regrouped into a

716 calculated module based on the network algorithm. 


\section{Fig. 1}
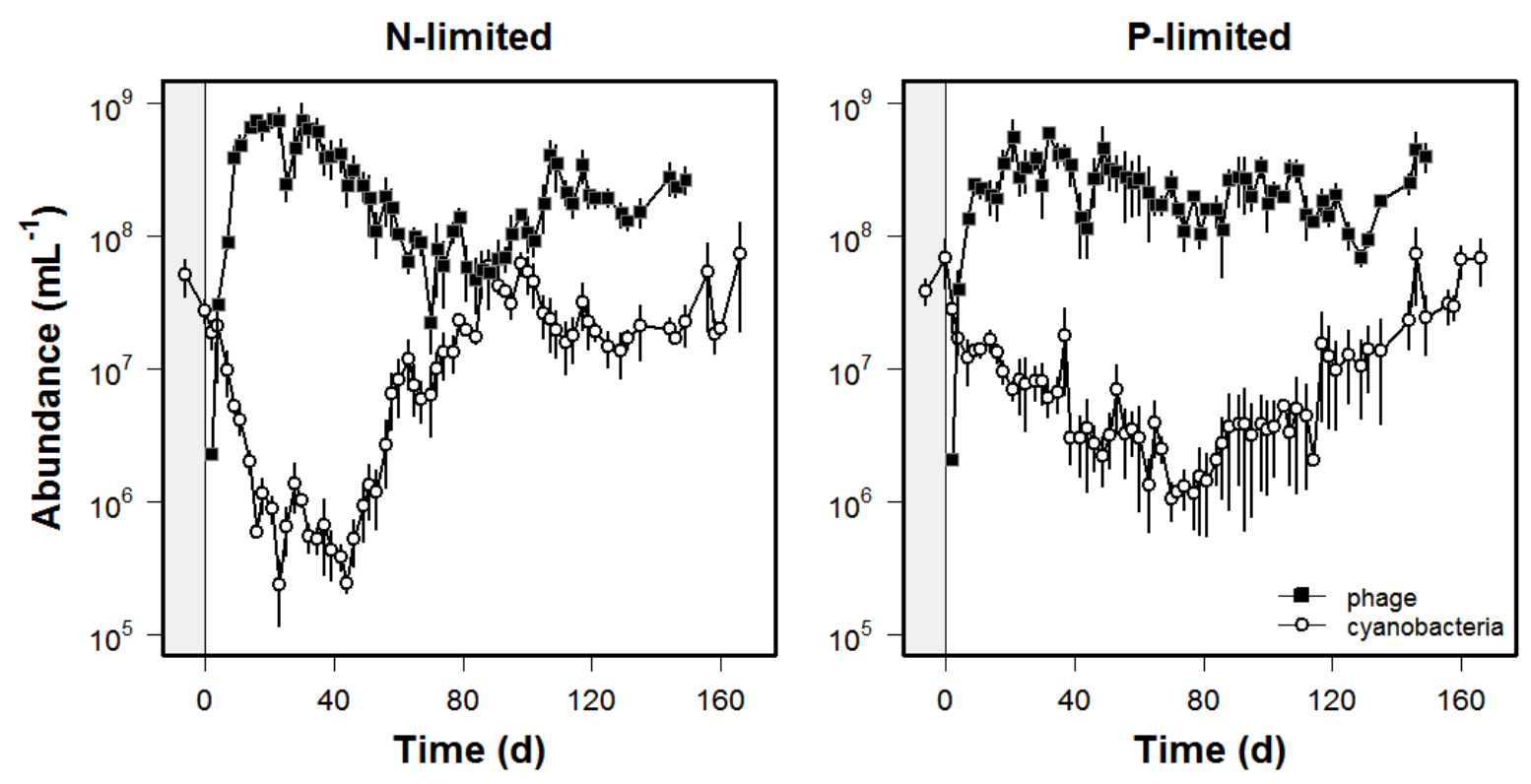
722

Fig. 2

724
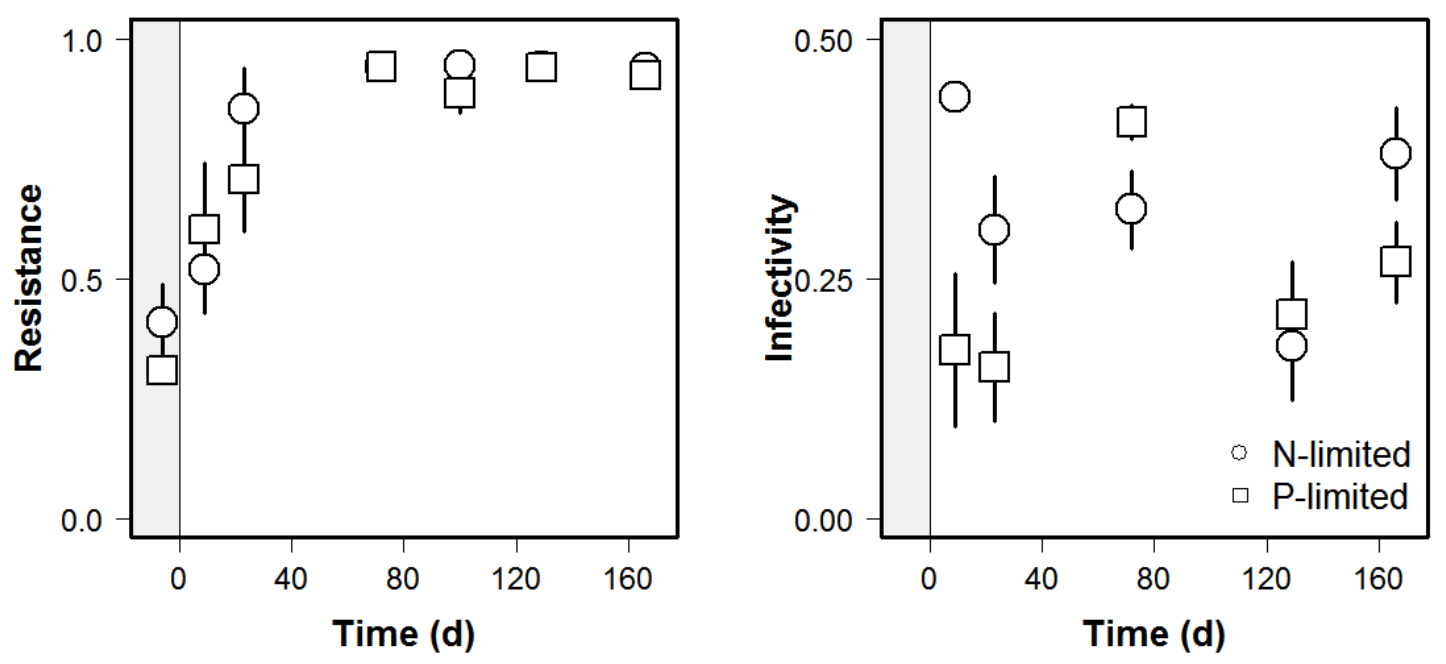

726 
Fig. 3
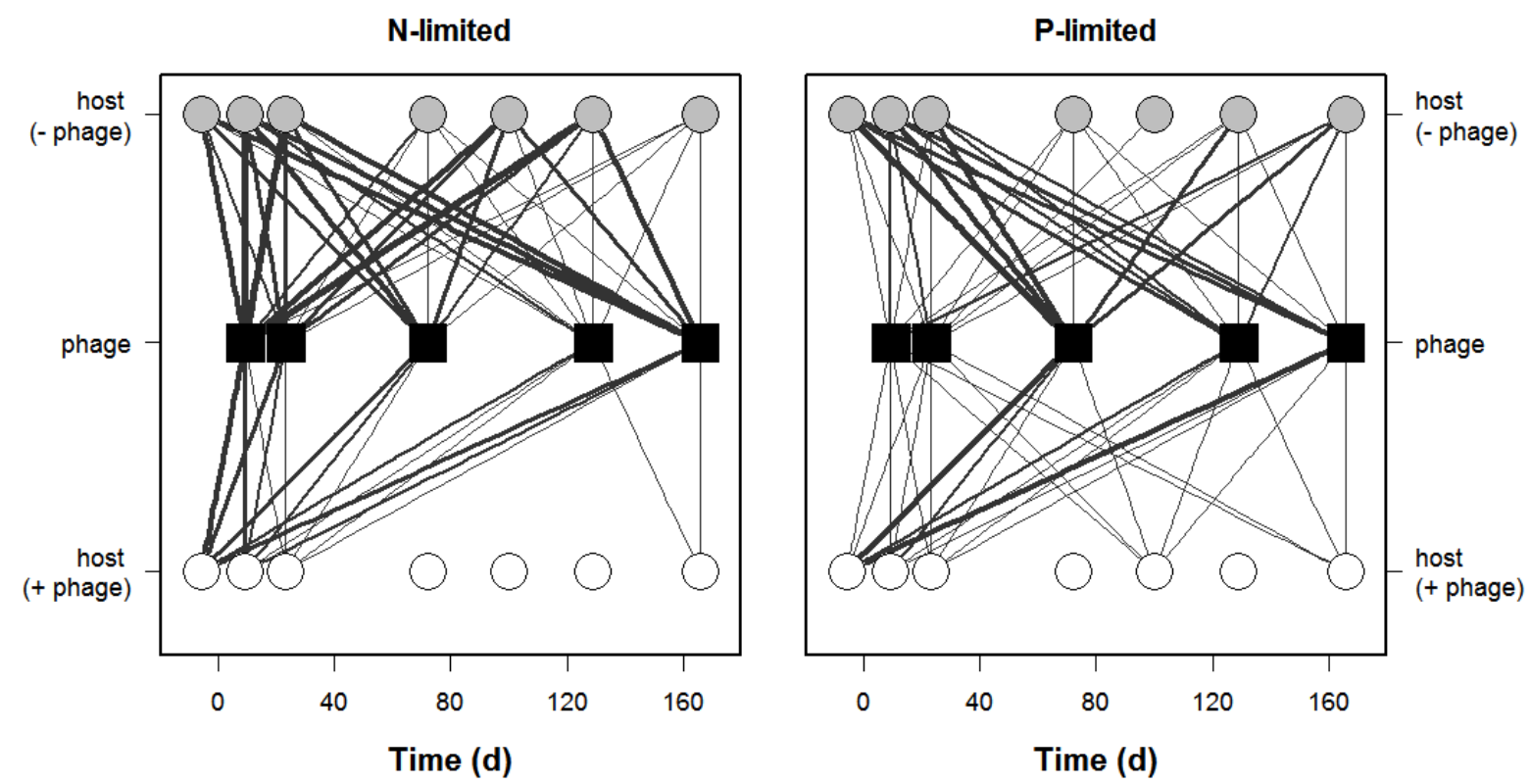
Fig. 4
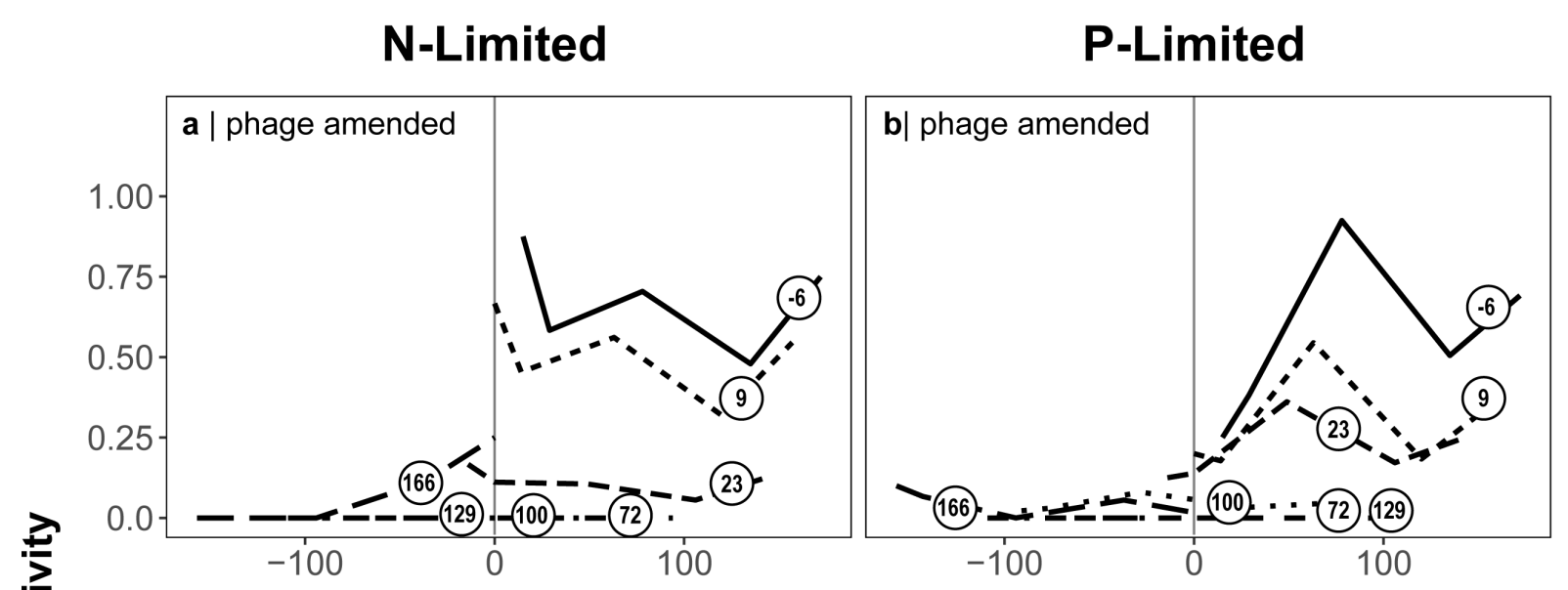

든

Time Shift (days) 
Fig. 5

742
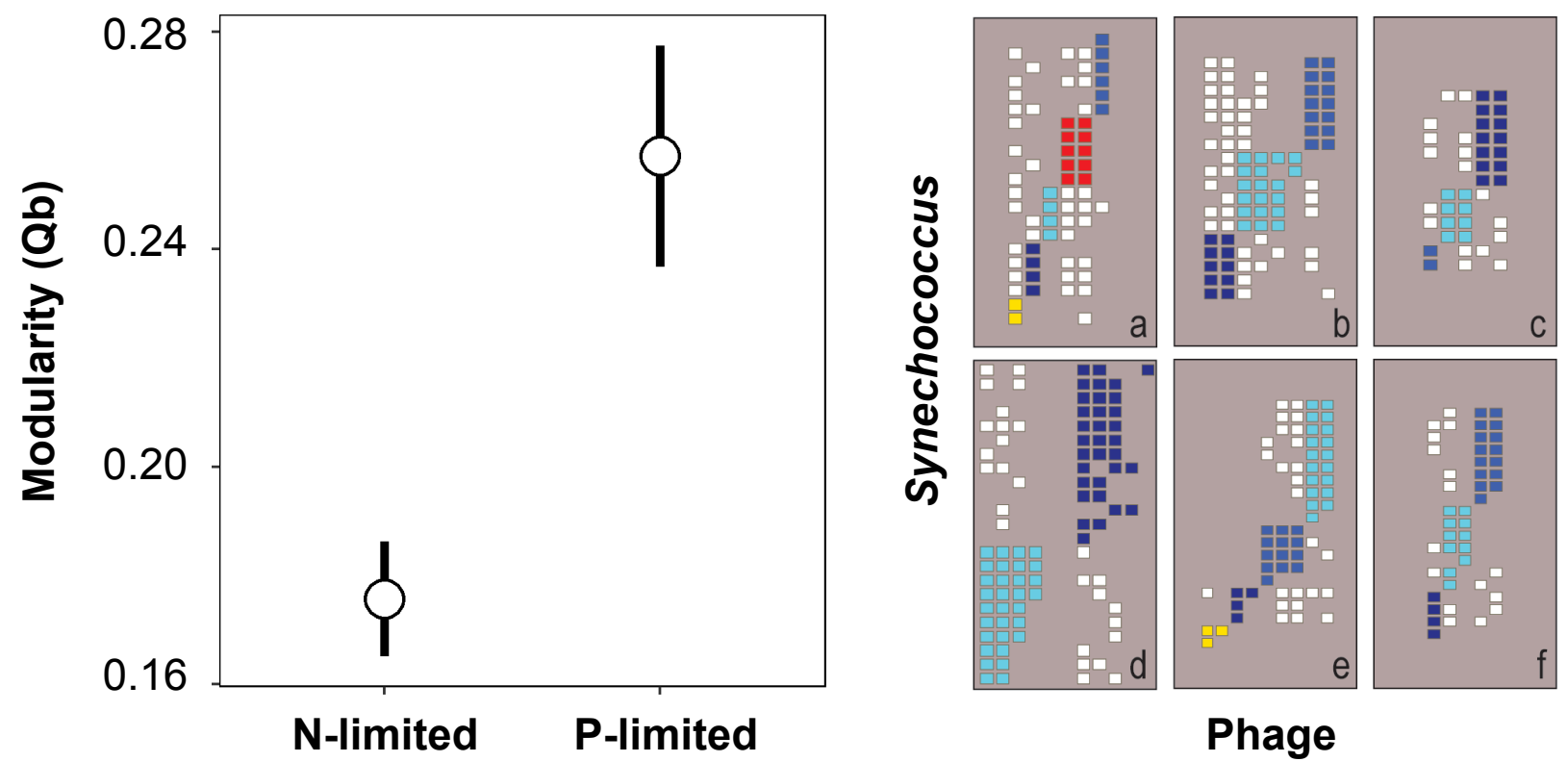

Phage

744

746 